\title{
Maturation Pathways of Dendritic Cells Determine TAP1 and TAP2 Levels and Cross-presenting Function
}

\author{
Andrés López-Albaitero ${ }^{*}$, Robbie Mailliard $\dagger$, Trevor Hackman ${ }^{*}$, Pedro A. Andrade Filho ${ }^{*}$ \\ Xinhui Wang ${ }^{\ddagger}$, William Gooding $\$$, Soldano Ferrone ${ }^{\ddagger}$, Pawel Kalinski ${ }^{\dagger}$, and Robert $L$. \\ Ferris ${ }^{*}, \|$ \\ "Department of Otolaryngology, University of Pittsburgh Cancer Institute, Pittsburgh, PA \\ tDepartment of Surgery, University of Pittsburgh Cancer Institute, Pittsburgh, PA \\ ‡Department of Pathology, University of Pittsburgh Cancer Institute, Pittsburgh, PA \\ "Department of Immunology, University of Pittsburgh Cancer Institute, Pittsburgh, PA \\ $\S$ Department of Biostatistics Facility, University of Pittsburgh Cancer Institute, Pittsburgh, PA
}

\section{Summary}

\begin{abstract}
Ability to cross-present exogenous antigens in the human leukocyte antigen class I pathway is key to the antigen presenting function of mature tumor cell-loaded dendritic cells (DC). Conditions of DC maturation have been shown to be important for DCs ability to produce proinflammatory cytokines and induce $\mathrm{T}$ cell effector functions. However, it remains unknown if the different pathways of maturation are associated with modulation of the ability of mature DCs to crosspresent tumor antigens (TA). Here, we compare DC matured with 3 clinically relevant cytokine combinations including interleukin (IL)- $1 \beta$, tumor necrosis factor- $\alpha$, IL-6 (termed DC-0), DC-0 cells incubated with prostaglandin-2 (termed DC-0+prostaglandin-2), or DC treated with interferon- $\gamma$, interferon- $\alpha$, tumor necrosis factor- $\alpha$, Poly I:C, and IL1- $\beta$ (termed DC- 1 ). We found that these DC vary in their ability to cross-present TA to cytotoxic T lymphocytes (CTL), with the DC- 1 cytokine combination being significantly more effective than the other 2 . TA cross presentation and CTL priming were strongly correlated with level of expression of the antigen processing machinery components, TAP1 and TAP2, indicating that these components could be used as biomarkers to standardize DC preparations for optimal function. However, the upregulation of TAP1/TAP2 was not sufficient to explain the enhanced cross-presentation ability of DC- 1 cells, as the use of IFN- $\gamma$ alone to up-regulate TAP1/TAP2 did not generate DC as effective at cross-presentation as the full DC-1 maturation cytokine combination. These data indicate for the first time that the pathways of DC maturation modulate antigen processing machinery component expression to different extents and that differently matured DC vary in the ability to cross-present TA to human leukocyte antigen class I-restricted CTL.
\end{abstract}

\section{Keywords}

antigen processing/presentation; dendritic cells; vaccination

Copyright ( 2009 by Lippincott Williams \& Wilkins

Reprints: Robert L. Ferris, The Hillman Cancer Center Research Pavilion, 5117 Centre Avenue, Room 2.26b, Pittsburgh, PA 15213 (ferrisrl@upmc.edu)..

Financial Disclosure: All authors have declared there are no financial conflicts of interest in regards to this work. 
Because of their high efficiency as antigen presenting cells, mature dendritic cells (DC) pulsed with antigen are frequently used as components of vaccines to present human leukocyte antigen (HLA) class I antigen-peptide complexes to cytotoxic T lymphocytes (CTL). When a minimal peptide epitope is used, no additional processing of the peptide is required to generate HLA class I antigen-peptide complexes. In contrast, the generation of such complexes from a protein or polypeptide requires endocytosis and processing by the antigen processing machinery (APM). Specifically, peptides generated by the proteasome from mostly, although not exclusively, endogenous proteins are transported into the endoplasmic reticulum by the transporter associated with antigen processing (TAP) subunits, TAP1 and TAP2. Here the TAP1/TAP2 complex is brought by tapasin into association with $\beta_{2}$ microglobulin-HLA class I heavy chain complexes, which are properly folded with the assistance of the chaperones, BiP, calnexin, calreticulin, and endoplasmic reticulump57. Trimeric HLA class I heavy chain- $\beta_{2}$ microglobulin-peptide complexes are then transported to the cell surface, where they are recognized by $\mathrm{T}$ cells expressing cognate $\mathrm{T}$ cell receptors (TCR). TCR signals transmitted by interaction with HLA class I antigen-peptide complexes, with the aid of costimulatory molecules, prime naive T cells, and a cellular immune response is induced.

The role of maturation stage of DC in their ability to present antigens to $\mathrm{T}$ cells has stimulated the development of a number of protocols to induce optimal maturation of DC. All of the available protocols that use different combinations of cytokines have been shown to induce the expression of cell surface markers and costimulatory molecules, such as CD40, CD80, CD83, CD86, CCR7, and HLA class II antigens, and promote the secretion of cytokines. It is generally accepted that expression of these surface markers is essential to the functional capacity of mature DC (mDC) to activate antigen specific CTL, although there is not a strict correlation between a single, particular marker and efficiency of DC priming of CTL. Furthermore to the best of our knowledge, no information is available about the ability of the various protocols to modulate the expression of APM components in spite of the crucial role played by this machinery in the generation of HLA class I-peptide complexes. As the lack of this information has a negative impact on our ability to optimize the maturation protocol and to standardize DC preparation, in this study we tested whether the level of APM components expressed by DC is influenced by the maturation protocol and whether these correlate with their ability to cross present TA to CTL.

\section{MATERIALS AND METHODS}

\section{Cell Lines}

The MAGE- $3^{+}$, HLA-A $* 0201^{-}$SCCHN cell line JHU-029, and MART- ${ }^{+}$, HLA-A*0201 ${ }^{+}$ human melanoma cell line FEM- $X^{1}$ were cultured in Dulbecco Modified Eagle Medium supplemented with $8 \%$ fetal bovine serum (Mediatech, Herndon, VA), 2\% L-glutamine, and $1 \%$ penicillin/streptomycin (Invitrogen Corp, Carlsbad, CA). For cross-presentation experiments the tumor cell lines were harvested from culture flasks using a trypsin/EDTA solution, resuspended in phosphate buffer-saline at the concentration of $1 \times 10^{6}$ cells $/ \mathrm{mL}$ and exposed to a $90 \mathrm{~W}, 312 \mathrm{~nm}$ UV-B light source (Fisher Scientific, Pittsburgh, PA) for 5 minutes to induce apoptosis. The mutant $\mathrm{T} 2$ cell line, which does not express low molecular weight protein (LMP) 2, LMP7, TAP1, and TAP2 gene products, ${ }^{2}$ was maintained in serum free medium AIM-V (Invitrogen Corporation). Cell lines and cultures were monitored for mycoplasma infection at monthly intervals. 


\section{Cytokines}

Granulocyte macrophage colony stimulating factor (GM-CSF), IFN- $\alpha$, IFN- $\gamma$, IL-1 $\beta$, IL-2, IL-4, IL-6, IL-7, PGE-2, Poly I:C, and TNF- $\alpha$ were purchased from R and D Systems (Minneapolis, MN). IFN- $\gamma$ was purchased from Intermune (Brisbane, CA).

\section{Antibodies}

HLA-A, HLA-B, HLA-C antigen-specific monoclonal antibodies (mAb) W6/32, HLA-A2, and HLA-A68 antigen-specific mAb BB7.2 $\delta$-specific mAb SY-4, MB-1-specific mAb SJJ-3, LMP 2-specific mAb SY-1, LMP10-specific mAb TO-7, TAP1-specific mAb NOB-1, TAP2-specific mAb NOB-2, tapasin-specific mAb TO-3 and HLA-DR antigenspecific mAb L243 were developed and characterized as described. ${ }^{3-10}$ FITC-labeled irrelevant specificity IgG1, FITC-labeled anti-CD80, FITC-labeled anti-CD83, FITC-labeled anti-CD86, and FITC-labeled anti-CCR7 mAb were purchased from BD Biosciences (San Jose, CA). The IFN- $\gamma$-specific mAb 1-D1 K and the IFN- $\gamma$-specific biotinylated mAb 7B6-1 were purchased from Mabtech (Mariemont, OH). The human IL-12 specific mAb (clone 249109) was purchased from R and D Systems (Minneapolis, MN). The avidinperoxidase complex, Vectastain Elite, and AEC Substrate were purchased from Vector labs (Burlingame, CA).

\section{Peptide and Tetramer}

The MAGE-3 $271-279$ (FLWGPRALV) ${ }^{11}$ and HIV POL $476-484$ (ILKEPVHGV) peptides were produced by the Peptide Synthesis facility at the University of Pittsburgh, using F-moc technology. Lyophilized peptides were resuspended at $1 \mathrm{mg} / \mathrm{mL}$ of dimethyl sulfoxide and used at the indicated concentrations for in vitro stimulation (IVS) of T cells and ELISPOT assays. The HLA-A*0201-MAGE-3 $271-279$ tetramer was synthesized by the NIH Tetramer Facility (Emory University, Atlanta, GA).

\section{Induction and Maturation}

Peripheral blood mononuclear cells (PBMCs) were obtained from the Western Pennsylvania Blood Bank in the form of buffy coat lymphocytes from a healthy individual. Fresh PBMC from these buffy coats were purified using a Ficoll-hypaque gradient (Amersham Biosciences). After typing for HLA-A*0201, monocytes were isolated from PBMC by plastic adherence for 2 hours at $37^{\circ} \mathrm{C}$. Plastic adherent cells were incubated at $37^{\circ} \mathrm{C}$ using Iscove modified Dulbecco medium (IMDM) media supplemented with $10 \%$ fetal bovine serum, GM-CSF (1000 IU/mL) and IL-4 (1000 IU/mL). On day 3 of the culture, GM-CSF, and IL-4 were replenished to a final concentration of 2000 and $1000 \mathrm{IU} / \mathrm{mL}$, respectively. Day 6 monocyte-derived, immature DC were cultured with the different cytokine combinations described in Table 1.

\section{Flow Cytometry}

Cell surface and intracytoplasmic staining with $\mathrm{mAb}$ were performed as described previously. ${ }^{9}, 12$ Mouse IgG1 mAb (Dako Laboratories, Carpinteria, CA) was used as a negative control and was adjusted to provide a standard mean fluorescence intensity (MFI) of 5 on the FL1 channel for intracellular and for cell surface staining, on a Coulter Beckman EPICS XL cytometer. The flow cytometer was calibrated daily using fluorescent beads by the flow cytometry facility personnel; this allowed comparison among all the experiments. All samples were run using identical settings collecting $\geq 10,000$-gated events. Analysis was performed using EXPO32 ADC software (Beckman-Coulter), expressing ratio of experimental versus control MFI or percentage of events shifting into (or out of) a defined gate. Surface markers and APM component expression was scored based on MFI as well as on percent positive cells (shifting into a defined flow cytometry gate). 


\section{Generation of TA-specific CTL by IVS}

HLA-A*0201-MAGE-3271-279-specific T cells (MAGE-specific CTL) were generated from HLA-A $2^{+}$healthy donor PBMC by IVS, as described previously, ${ }^{13-15}$ with the following modifications. Monocytes were cultured for 6 days in IMDM medium (Mediatech, Herndon, VA) supplemented with GM-CSF and IL-4, each at the final concentration of $1000 \mathrm{IU} / \mathrm{mL}$. The resulting immature DC were incubated for 48 hours at $37^{\circ} \mathrm{C}$ with IFN- $\alpha(1000 \mathrm{U} / \mathrm{mL})$, IFN- $\gamma(1000 \mathrm{U} / \mathrm{mL}), \mathrm{IL}-1 \beta(25 \mathrm{ng} / \mathrm{mL})$, and TNF- $\alpha(50 \mathrm{ng} / \mathrm{mL})$.

Cells were incubated with exogenous peptide $(1 \mu \mathrm{M})$ for 2 hours at $37^{\circ} \mathrm{C}$, then mixed with naive $\mathrm{CD} 8^{+}$cells isolated by negative selection from nonadherent PBMC using MACS beads (Miltenyi Biotec) at a ratio of 1:10. Cells were resuspended in IMDM medium supplemented with IL-2 (50 ng/mL), IL-7 (25 ng/mL), and $2 \%$ human AB serum. CTL were expanded in vitro and stimulated every 2 weeks using irradiated T2 cells pulsed with exogenous TA-derived peptide. Specificity of the CTL was determined with the use of HLA-A*0201:MAGE-3271-279 tetramer staining and IFN- $\gamma$ ELISPOT.

MART-127-35 specific CTL were generated by IVS in the same fashion as MAGE-3 specific CTL, however, instead of exogenous peptide DC were pulsed with apoptotic FEM$\mathrm{X}$ cells (as detailed above).

\section{ELISPOT Assays}

IFN- $\gamma$ ELISPOT assays were performed, as described previously, ${ }^{15}$ using as targets monocyte-derived, mDC pulsed with TA-derived peptide or apoptotic tumor cells. All experiments used HLA-A*0201-MAGE-3271-279-specific CTL and T2 mutant cells pulsed for 2 hours at room temperature with $1 \mu \mathrm{M}$ of MAGE-3271-279 or Influenza Matrix ${ }_{58-66}$ peptide. HLA class I antigen-restriction of T cells was tested by incubating target cells with HLA class I antigen-specific mAb W6/32 at $10 \mu \mathrm{g} / \mathrm{mL}$, for 30 minutes at $37^{\circ} \mathrm{C}$ before being used as targets in cytotoxicity assays. HLA-DR antigen-specific mAb L243, at $10 \mu \mathrm{g} / \mathrm{mL}$, was used as a specificity control. Plates were read using a Carl Zeiss VISION ELISPOT reader. Background was considered as the number of spots produced by the $\mathrm{CD} 8^{+} \mathrm{T}$ cells alone.

\section{Statistical Analyses}

Broad equality of APM component expression by Day 8 DC and by DC matured with the 3 cytokine combinations was tested with the Kruskal-Wallis test. If this test produced $P<0.05$ then Day 8 DCs were compared with all maturation regimens with the Wilcoxon test. DC matured with the 3 cytokine combinations were compared with each other and also with the Wilcoxon test. All tests were 2 sided and test statistics were based upon the exact permutation distribution of the test statistic. CTL responses in IFN- $\gamma$ ELISPOT assays were scored as positive if a 1 tailed permutation test that compared test wells to background wells produced a $P$ value $\leq 0.05$.

\section{RESULTS}

\section{Similar Surface Phenotype of DC Matured With Different Cytokine Combinations}

Table 1 lists the combinations of cytokines used. DC generated after culture with GM-CSF and IL-4 for 6 days were then exposed for 48 hours at $37^{\circ} \mathrm{C}$ to the maturation cytokine combinations listed in Table 1. Day 8 DC, used as a baseline for comparison, was incubated under the same experimental conditions but was not exposed to cytokines between day 6 and 8. MDC, generated by incubation of DC with IL- $1 \beta$, IL- 6 , and TNF- $\alpha$ were termed DC-0. DC generated by incubating DC with the same combination of cytokines used for DC- 0 except for the addition of PGE- 2 were termed DC-0+PGE-2. A third set of $\mathrm{mDC}$ were 
generated by incubating DC with IFN- $\alpha$, IFN- $\gamma$, IL-1 $\beta$, Poly I:C, and TNF- $\alpha$, and were termed DC-1 (Table 1). As shown in Figure 1, all the cytokine combinations used induced similar expression levels of HLA class I and class II antigens and of CD83, CD86, and CCR7 indicating a similar state of surface phenotypic maturation.

\section{Effect of Different Cytokine Combinations on APM Component Expression by DC}

The baseline level of expression of HLA class I pathway APM components, was determined by cytofluoro-graphic analysis of DC incubated for 8 days without maturation cytokine exposure, then intracellular staining with APM component specific mAb. Representative results obtained from 1 experiment are shown in Figure 2A. The MFI for HLA antigens and APM components in DC generated from PBMC obtained from 12 healthy donors is summarized in Figure 2B. Day 8 DC was characterized by a low level of expression of all the APM components. Some of the components, such as LMP2 were barely detectable. We first noted that incubation of DC with all of the cytokine combinations enhanced the expression of all the APM components. When the 3 cytokine combinations were simultaneously compared with Day 8 DCs, APM components TAP1 and TAP2, and tapasin were up-regulated $(P=0.0003,0.0002$, and 0.0560 , respectively, (Table 2). Among the 3cytokine combinations used, the DC-1 combination was more effective than either DC-0 or DC-0+PGE-2 in up-regulation of TAP1 and TAP2. No significant differences were observed between DC-0 and DC-0+PGE-2 combinations for any of the other APM components studied (LMP2, LMP10, MB1, and $\delta$ ). These results are summarized in Table 2 and Figure 2B.

\section{Marked Up-regulation by IFN-y of TAP1 and TAP2 Expression in Mature DC}

To identify the cytokine(s) in the DC-1 cytokine combination responsible for enhancement of TAP1 and TAP2 expression, we compared the changes in APM component expression in DC incubated with each cytokine individually versus DC incubated with the complete DC-1 cytokine, using expression by DC as a baseline. Figure 3 shows a representative experiment, repeated three times for each cytokine or cytokine combination. Changes in APM component expression in DC after incubation with IFN- $\gamma$ alone were comparable to DC matured with the complete DC-1 cytokine combination. However, the level of expression of surface markers, CD80, CD83, CD86 including CCR7, by DC treated with IFN- $\gamma$ was lower than that expressed by DC-1 (data not shown). These results are compatible with a key role for IFN- $\gamma$ in promoting high expression of APM components in DC-1 cells. Incubation of DC with Poly I:C, TNF- $\alpha$, IL-1 $\beta$, or IFN- $\alpha$ alone did not up-regulate APM component expression significantly (not shown).

\section{Correlation of TAP1 and TAP2 Expression With Cross-presentation Efficiency by Mature DC}

To assess the functional significance of the differential expression of APM components in DC treated with the 3 different cytokine combinations, DC were tested for their ability to cross-present the MAGE-3271-279 peptide from apoptotic HLA-A2 ${ }^{-}$, MAGE- $3^{+}$tumor cells to HLA-A2 restricted, MAGE-specific CTL. MAGE-3271-279 specific CTL were tested after each stimulation for specificity as detailed in the Materials and Methods section (Fig. 4A). DC were cocultured for 48 hour at $37^{\circ} \mathrm{C}$, during the phase of exposure to each cytokine combination, with apoptotic MAGE- $3^{+}$, HLA-A2 ${ }^{-}$JHU-029 cells as a source of antigen. These DC were then tested for recognition by MAGE-specific CTL using IFN- $\gamma$ ELISPOT assays. Controls using the MAGE- $3^{-}$HLA-A2 ${ }^{+}$SCC4 cells and the HLA-A, HLA-B, HLA$\mathrm{C}$ specific $\mathrm{mAb}$ W6/32 were included to establish MAGE-3 specific, HLA class I restricted activity (Fig. 4B). Mature and Day 8 DC was tested in parallel for surface marker and intracellular APM component expression. The expression of these markers was then correlated with recognition by MAGE-CTL using IFN- $\gamma$ ELISPOT assays (Fig. 4B). 
Although all DC were capable of some degree of cross-presentation, most efficient presentation to MAGE-CTL occurred when DC-1 were used to cross-present MAGE-3 $271-279$. Nonspecific, background CTL activation was observed with DC fed with MAGE- $3^{-}$HLA-A2 ${ }^{+}$SCC 4 cells which was significantly lower to CTL activation observed after DC were incubated with MAGE- $3^{+}$apoptotic cells (Fig. 4B). Statistical analysis of APM component expression indicated that only TAP1 $(P=0.011)$ and TAP2 $(P=0.036)$ expression levels were correlated with the best cross-presenting DC-1 maturation phenotype with tapasin expression approaching statistical significance $(P=0.0693)$.

To exclude that differences observed in cross presentation were a result of increased IL-12 secretion, a well known characteristic the DC-1 phenotype, ${ }^{14}$ apoptotic JHU-029 tumor pulsed DC-1 cells were incubated with a neutralizing IL-12 specific mAb $(10 \mu \mathrm{g} / \mathrm{mL}$ for 18 h). No significant reduction in cross presentation to the MAGE-CTL clone was observed (Fig. 4C) excluding a role for IL-12 secretion to explain the efficiency of DC-1 crosspresentation.

Likewise, to exclude that differences observed in cross presentation reflected differences in cytokines or costimulatory molecules, all DC types were pulsed with exogenous MAGE-3 $271-279$ peptide (10 $\mu \mathrm{g} / \mathrm{mL}$ for $1 \mathrm{~h}$ at room temperature) and tested for recognition using MAGE-3 specific CTL using IFN- $\gamma$ ELISPOT assays (Fig. 4D). All MAGE-3271-279 peptide pulsed DC were recognized by MAGE-CTL. However, recognition of both MAGE-3 $271-279$ peptide pulsed DC-1 and DC-0+PGE-2 was significantly higher than either Day 8 DC or DC-0 $(P<0.05)$. Despite the similar recognition of peptide pulsed DC-0+PGE-2 and the higher expressing TAP1/TAP2 DC-1 cells, the latter were significantly better cross-presenters to MAGE-3 specific CTL.

Cross-presenting ability was not robust in DC treated with IFN- $\gamma$ alone, likely owing to the lower expression of surface costimulatory molecules as described above (data not shown), suggesting that IFN- $\gamma$-induced TAP1/2 up-regulation alone is not sufficient to confer the strong cross-presenting ability observed in DC-1 cells.

\section{Stimulation of Resting T Cells by DC-1 Cross-presentation}

To test whether the differentially matured DCs loaded with tumor cell-derived antigens would also differ in the induction of TA-specific CTL responses from naive PBMC, we evaluated the ability of apoptotic MART-1 ${ }^{+}$tumor cell loaded DC-1 to induce MART-1specific CTL responses. Because no significant differences were observed in previous experiments between DC-0 and DC-0+PGE-2, the DC-0+PGE- 2 cytokine combination was used for these experiments and compared with Day 8 DC or DC-1 cells. For stimulation of naive T cells from PBMC, only DC-1 but not Day 8 DC or DC-0+PGE-2, proved to be effective in inducing MART-1-specific CTL (Fig. 5). The DC-1 primed CTL could efficiently recognize the parental FEM-X melanoma cells (Fig. 5A) or MART-1 27-35 peptide pulsed T2 cells (Fig. 5B). These results indicate that the enhanced cross-presentation by DC-1 occurs both with resting T cells and effector CTL.

\section{DISCUSSION}

In this study, we demonstrate that the conditions of DC maturation differentially influence the expression of APM components and the ability to cross-present tumor-derived antigens to HLA class I-restricted CTL. Although several APM components were modulated in their expression, we found that only selected APM components, TAP1 and TAP2, were significantly correlated with cross-presentation to T cells, being particularly high in the cells matured with the DC-1 cytokine combination (Table 1). A number of other factors, besides expression of APM components TAP1 and TAP2, could be responsible for this capacity, 
including surface costimulatory markers, adhesion molecules, and stimulatory cytokines. However we observed no significant differences in expression of CD80, CD83, CD84, CD40 or CCR7 between the different mDC. Additionally, the results observed in CTL recognition of tumor fed DC differed with those observed with DC pulsed with exogenous peptide. Furthermore, MAGE-3 specific CTL recognition was not affected by an IL-12 blocking $\mathrm{mAb}$, demonstrating that differences in cross-presentation are more likely a consequence of a different intracellular phenotype, owing to differences in APM component expression rather than differences in cell surface markers or secretion of IL-12 or other cytokines. Furthermore, whereas we and others ${ }^{16,17}$ have previously demonstrated that the ability of DC to produce IL-12 is important for their ability to induce TA-specific functional CTL responses from resting T cells, ${ }^{14}$ the current findings indicate that that this cytokine is not essential for the ability of DC to cross-present antigen to already primed, functional CTL.

Because of the strong correlation of TAP1 and TAP2 expression with DC stimulatory capacity, we studied the importance of selective APM component up-regulation in $\mathrm{mDC}$, through maturation with IFN- $\gamma$ alone. However, despite the marked up-regulation of TAP1/ TAP 2 by IFN- $\gamma$ similar to that seen using the most stimulatory DC- 1 cytokine combination (Fig. 3) DC treated with IFN- $\gamma$ alone had poor stimulatory capacity and cross-presentation of a MAGE-3-derived peptide was not robust in IFN- $\gamma$ matured cells, suggesting that other factors are necessary to achieve the full DC function desired for cancer vaccines, including antigen uptake and surface costimulation. This result also implies that TAP1/2 provide only some of the properties of DC necessary for optimal activity to generate and maintain CTL responses. Similarly, it has been demonstrated that IFN- $\gamma$ used in conjunction with other maturation stimuli (such as lippopolysaccharide), promotes CCR7-driven migration, IL-12p and IL-27 secretion, and decrease IL-10 production, but is unable to achieve these effects when used alone. ${ }^{18}$ Thus, our and other's findings, suggests that further studies should be conducted to investigate the value of incorporating IFN- $\gamma$ into DC maturation and T cell stimulation, and Th1 instruction, in vitro and in vivo.

Clinical vaccine trials incorporating TAP1 and TAP2 expression measurements may allow us to directly address the efficacy of this approach. Thus, we conclude that a complex maturation combination is necessary to fully mature DC, and that TAP $1 / 2$ expression alone is not sufficient to generate the most stimulatory DC for cancer vaccines. Irrespective of the mechanism underlying optimal cross-presentation, our results also suggest that monitoring of DC preparations for TAP1 and TAP2 expression may represent useful biomarkers to permit standardization of phenotypic and functional attributes for improved cancer vaccines.

For the first time, we also show the role of APM component expression is strongly related to the ability of DC to cross-present TA to naive and primed CTL in vitro. These findings are consistent with the observations of others, where transfection of selected APM components result in higher levels of cross-presentation and cross-priming in mice. ${ }^{19,20}$ If these results are borne out by further studies, including our identification of a putative marker to standardize DC, significant utility for optimizing and monitoring vaccine trials might be obtained. The potential to characterize DC by their intracellular, antigen processing phenotype according to APM component expression would enable consistent generation of DC most likely to generate antigen-specific immune responses in vaccines loaded with the tumor-related particulate material, rather than peptides. In addition, future studies may address whether APM components are involved in the processing of long peptides used in some recent vaccine regimens. ${ }^{21}$

The effect of DC maturation protocols may also facilitate access of exogenous TA into the HLA class I antigen-processing pathway, as this process begins with cytoplasmic 
degradation and is generally TAP $1 / 2$ dependent. ${ }^{22}$ We have shown that cross-presentation is strongly influenced by DC maturation phenotype, leading to alterations in TAP1/2 expression. It is possible that further studies will elucidate the effect of DC maturation pathways on facilitating access of antigen taken up exogenously into the cytoplasmic compartment, where interaction with these APM components can occur efficiently.

The most striking aspect of our finding was that DC, which express very similar levels of surface phenotypic markers of maturation, differed significantly in their ability to crosspresent exogenous antigen, Thus, it is possible that measuring TAP1 and TAP2 would be sufficient to indicate appropriately matured DC, as a direct measure of intracellular processing and presenting function for DC-based immunotherapy. We believe that these APM markers should be incorporated routinely into DC-based vaccines and in vitro studies which require comparable and full DC maturation between experiments. Additional studies should also validate these findings in vivo, by incorporating measurement of APM component expression into ongoing DC-based vaccine trials using tumor-derived antigenic material. Our data suggest that appropriate in vivo manipulation of $\mathrm{mDC}$ can yield cells capable of both efficient antigen processing as well as surface HLA-TA peptide specific stimulation of antigen-specific $\mathrm{T}$ cells.

\section{Acknowledgments}

This work was supported by R01 CA110249, P50 CA097190, and a FAMRI Clinical Investigator Award.

\section{REFERENCES}

1. Lin C, Grandis JR, Carey TE, et al. Lai Head and neck squamous cell carcinoma cell lines: established models and rationale for selection. Head and Neck 2007;29:163-188. [PubMed: 17312569]

2. Salter RD, Cresswell P. Impaired assembly and transport of HLA-A and HLA-B antigens in a mutant TxB cell hybrid. EMBO J 1986;5:943-949. [PubMed: 3522223]

3. Parham PBC, Bodmer WF. Use of a monoclonal antibody (W6/32) in structural studies of HLA-A, B, C, antigens. J Immunol 1979;123:342-349. [PubMed: 87477]

4. Parham PBW. Monoclonal antibody to a human histocompatibility alloantigen, HLA-A2. Nature 1978;276:397-399. [PubMed: 714164]

5. Lampson LA, Levy R. Two populations of Ia-like molecules on a human B cell line. J Immunol 1980;125:293-299. [PubMed: 6966655]

6. Bandoh N, Ogino T, Cho HS, et al. Development and characterization of human constitutive proteasome and immunoproteasome subunit-specific monoclonal antibodies. Tissue Antigens 2005;66:185-194. [PubMed: 16101829]

7. Barnstable CJ, Bodmer WF, Brown G, et al. Production of monoclonal antibodies to group A erythrocytes, HLA and other human cell surface antigens-new tools for genetic analysis. Cell 1978;14:9-20. [PubMed: 667938]

8. Temponi M, Kekish U, Hamby CV, et al. Characterization of anti-HLA class II monoclonal antibody LGII-612.14 reacting with formalin fixed tissues. J Immunol Methods 1993;161:239-256. [PubMed: 8505553]

9. Ogino T, Wang X, Kato S, et al. Endoplasmic reticulum chaperone-specific monoclonal antibodies for flow cytometry and immunohistochemical staining. Tissue Antigens 2003;62:385-393. [PubMed: 14617045]

10. Wang X, Campoli M, Cho HS, et al. A method to generate antigen-specific mAb capable of staining formalin-fixed, paraffin-embedded tissue sections. J Immunol Methods 2005;299:139_ 151. [PubMed: 15896802]

11. Gaugler B, Van den Eynde B, van der Bruggen P, et al. Human gene MAGE-3 codes for an antigen recognized on a melanoma by autologous cytolytic T lymphocytes. J Exp Med 1994;179:921-930. [PubMed: 8113684] 
12. Ogino T, Wang X, Ferrone S. Modified flow cytometry and cell-ELISA methodology to detect HLA class I antigen processing machinery components in cytoplasm and endoplasmic reticulum. J Immunol Methods 2003;278:33-44. [PubMed: 12957394]

13. Lopez-Albaitero A, Nayak JV, Ogino T, et al. Role of antigen-processing machinery in the in vitro resistance of squamous cell carcinoma of the head and neck cells to recognition by CTL. J Immunol 2006;176:3402-3409. [PubMed: 16517708]

14. Mailliard RB, Wankowicz-Kalinska A, Cai Q, et al. alpha-type-1 polarized dendritic cells: a novel immunization tool with optimized CTL-inducing activity. Cancer Res 2004;64:5934-5937. [PubMed: 15342370]

15. Sirianni N, Ha PK, Oelke M, et al. Effect of human papillomavirus-16 infection on CD8+ T-cell recognition of a wild-type sequence p53264-272 peptide in patients with squamous cell carcinoma of the head and neck. Clin Cancer Res 2004;10:6929-6937. [PubMed: 15501971]

16. Jonuleit H, Kuhn U, Muller G, et al. Pro-inflammatory cytokines and prostaglandins induce maturation of potent immunostimulatory dendritic cells under fetal calf serum-free conditions. Eur J Immunol 1997;27:3135-3142. [PubMed: 9464798]

17. Thumann P, Moc I, Humrich J, et al. Antigen loading of dendritic cells with whole tumor cell preparations. J Immunol Methods 2003;277:1-16. [PubMed: 12799035]

18. Frasca L, Nasso M, Spensieri F, et al. IFN-gamma arms human dendritic cells to perform multiple effector functions. J Immunol 2008;180:1471-1481. [PubMed: 18209042]

19. Lou Y, Basha G, Seipp RP, et al. Combining the antigen processing components tap and tapasin elicits enhanced tumor-free survival. Clin Cancer Res 2008;14:1494-1501. [PubMed: 18316574]

20. Vitalis TZ, Zhang QJ, Alimonti J, et al. Using the TAP component of the antigen-processing machinery as a molecular adjuvant. PLoS pathogens 2005;1:e36. [PubMed: 16389301]

21. Zwaveling S, Ferreira Mota SC, Nouta J, et al. Established human papillomavirus type 16expressing tumors are effectively eradicated following vaccination with long peptides. J Immunol 2002;169:350-358. [PubMed: 12077264]

22. Huang AY, Bruce AT, Pardoll DM, et al. In vivo cross-priming of MHC class I-restricted antigens requires the TAP transporter. Immunity 1996;4:349-355. [PubMed: 8612129] 

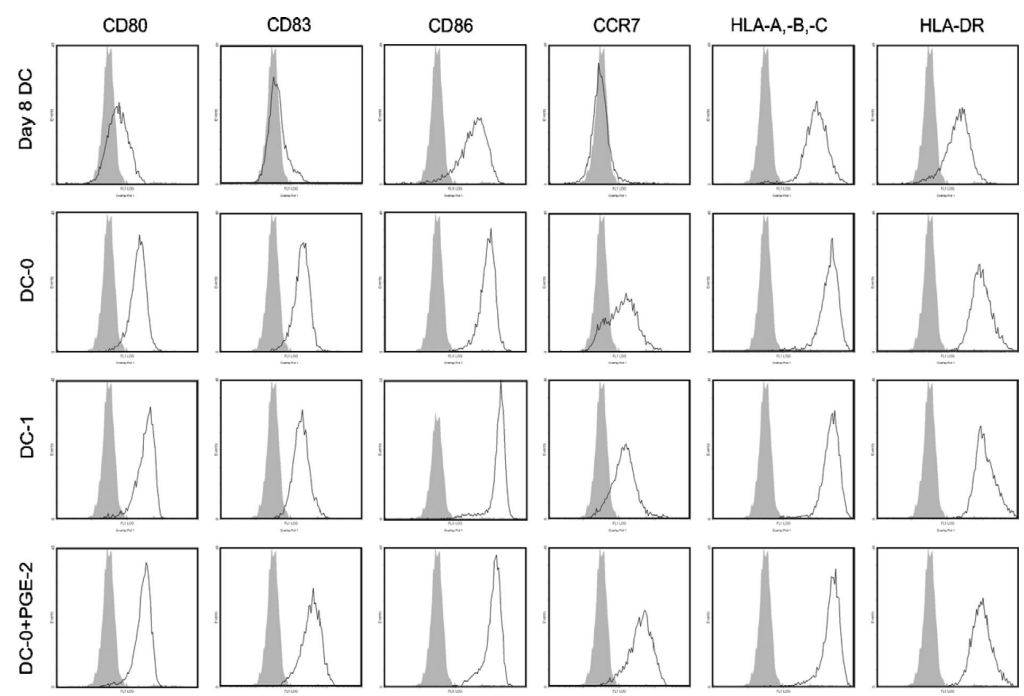

FIGURE 1.

Expression of costimulatory molecules by DC after incubation with different cytokine combinations. After six days of culture in interleukin- 4 and granulocyte macrophage colony stimulating factor supplemented media, human monocyte derived immature DC was incubated for $48 \mathrm{hr}$ at $37^{\circ} \mathrm{C}$ in medium alone or in medium supplemented with 1 of the maturation cytokine combinations (Table 1). Cells were then harvested and stained with FITC labeled control IgG (gray histograms), anti-CD80, anti-CD83, anti-CD86, anti-CCR7 or HLA-DR-specific monoclonal antibodies (solid lines). Samples were analyzed by flow cytometry, adjusting the mean fluorescence intensity for control IgG-stained cells to 5 to permit comparison between DC culture conditions. DC indicates dendritic cell; IgG, immunoglobulin G. 

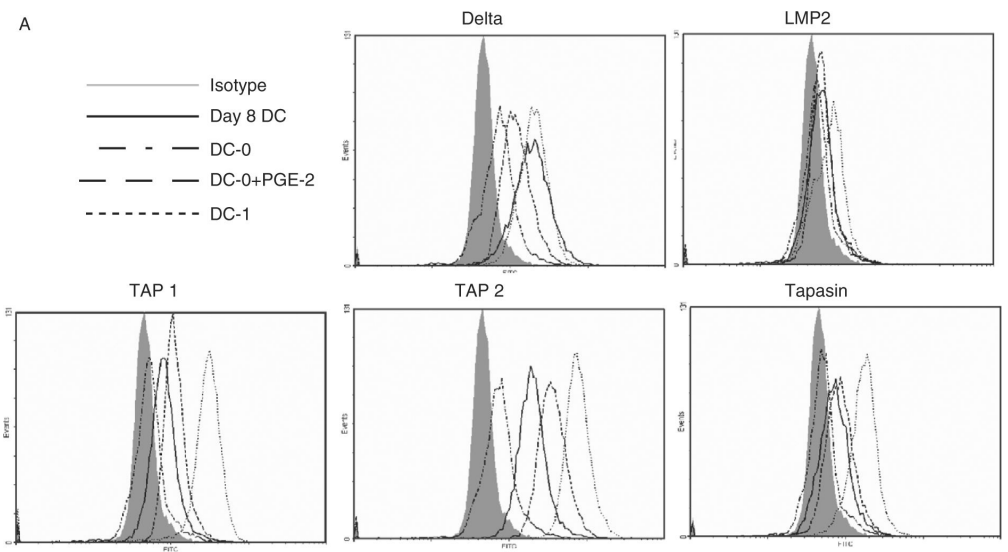

B

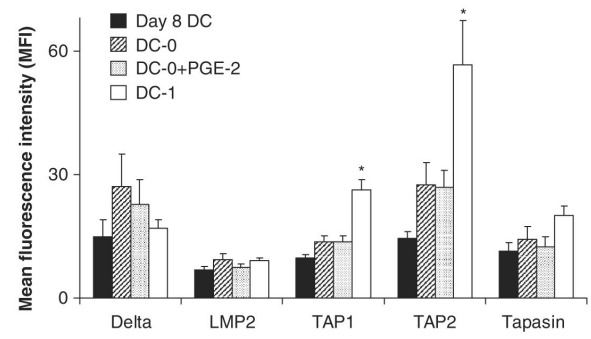

FIGURE 2.

Differential APM component up-regulation in DC matured with different cytokine combinations. A, representative flow cytometry histograms for APM component expression are shown, as performed for 12-healthy donor DC preparations. Separate experiments were repeated for 2 or 3 times per donor. After 6 days of culture in interleukin- 4 and granulocyte macrophage colony stimulating factor supplemented media, human monocyte-derived immature DC were treated with medium alone or medium cultured with 1 of the cytokine combinations listed in Table 1 for 48 hour at $37^{\circ} \mathrm{C}$. DC were then harvested and stained intracellularly with mAb specific for $\delta$, LMP2, TAP1, TAP2 or tapasin (as described in Materials and Methods section). Stained cells were analyzed by flow cytometry, adjusting the MFI for control isotype matched mAb-stained cells to 5, to permit comparison between different DC culture conditions. B, MFI values summarized from 12 sets of healthy, HLA$\mathrm{A}^{*} 0201^{+}$donor DC that were matured with different cytokine combinations listed in Table 1. Mean MFI values ( \pm SEM) obtained by flow cytometry are shown, where the isotype control staining was set to $\mathrm{MFI}=5$, to permit comparison between different DC culture conditions $(* P<0.05)$. APM indicates antigen processing machinery; DC, dendritic cell; $\mathrm{mAb}$, monoclonal antibody; MFI, mean fluorescence intensity. 


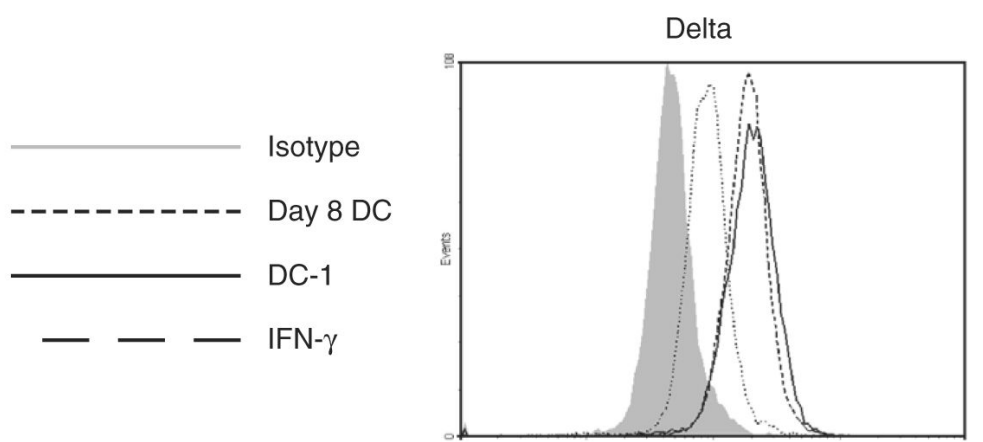

TAP1
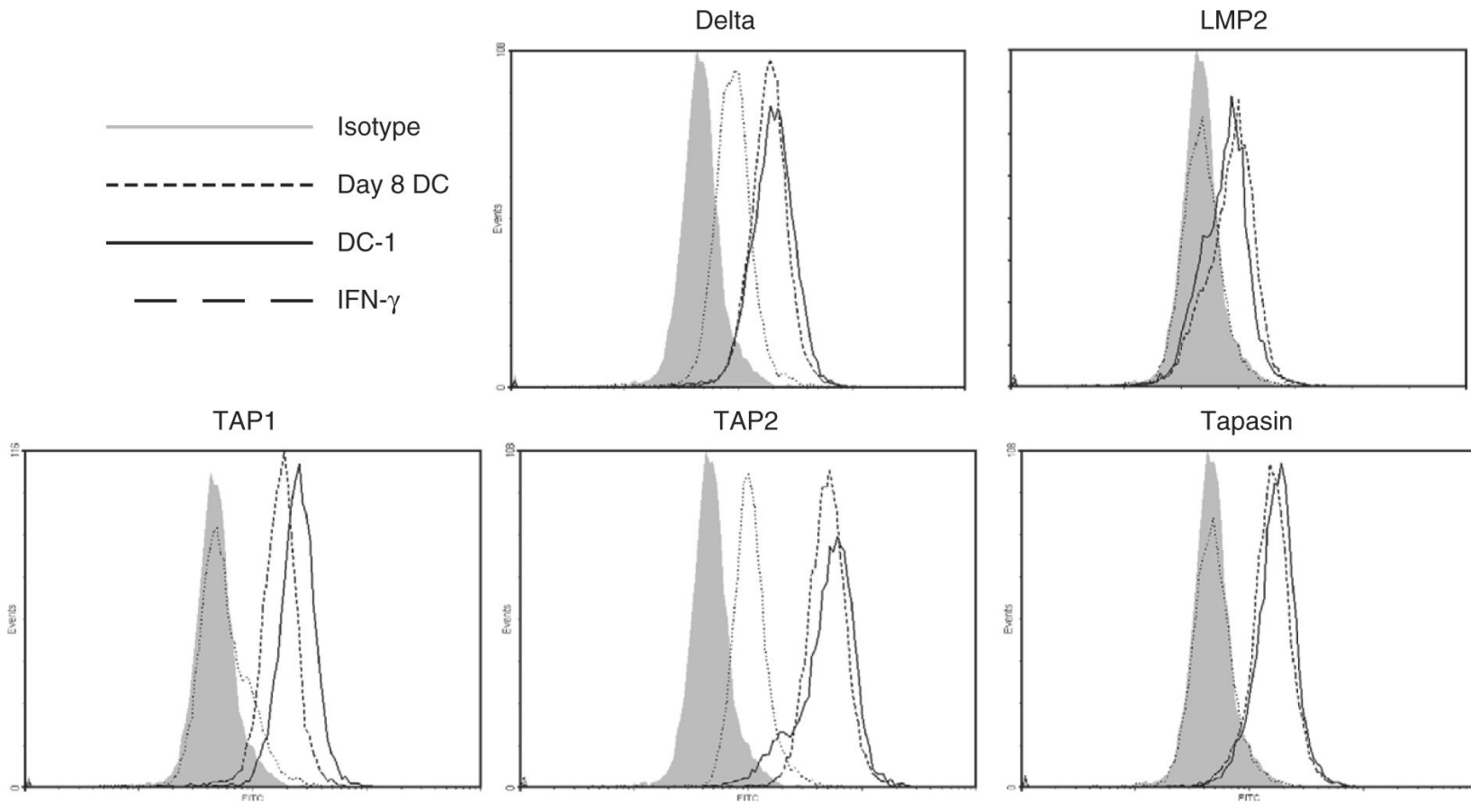

Tapasin

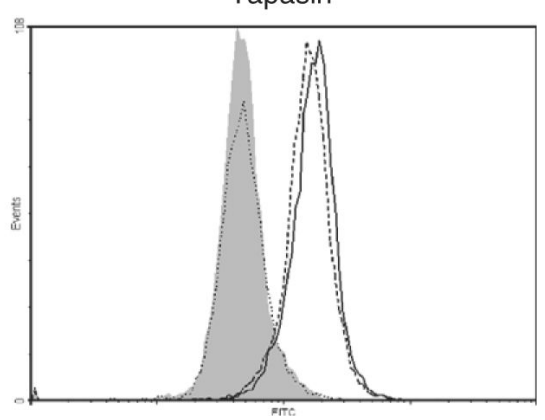

FIGURE 3.

Expression of antigen processing machinery components, $\delta$, LMP2, TAP1, TAP2, and tapasin, after treatment with IFN- $\gamma$ alone or with the DC- 1 cytokine combination. Immature DC were incubated with IFN- $\gamma$ alone $\left(100 \mathrm{IU} / \mathrm{mL}\right.$ for $48 \mathrm{~h}$ at $\left.37^{\circ} \mathrm{C}\right)$ or with the DC-1 cytokine combination (tumor necrosis factor- $\alpha$ at $50 \mathrm{ng} / \mathrm{mL}$, interleukin- $1 \beta$ at $25 \mathrm{ng} / \mathrm{mL}$, IFN- $\alpha$ at $1000 \mathrm{U} / \mathrm{mL}$, and IFN- $\gamma$ at $1000 \mathrm{U} / \mathrm{mL}$ for $48 \mathrm{~h}$ at $37^{\circ} \mathrm{C}$ ). DC were then harvested, stained intracellularly and analyzed by flow cytometry. Mean MFI values (+SEM) obtained from the flow cytometry analysis are shown. The isotype control monoclonal antibody staining was set to MFI $=5$ to permit comparison between different DC culture conditions. DC indicates dendritic cell; IFN, interferon; MFI, mean fluorescence intensity. 


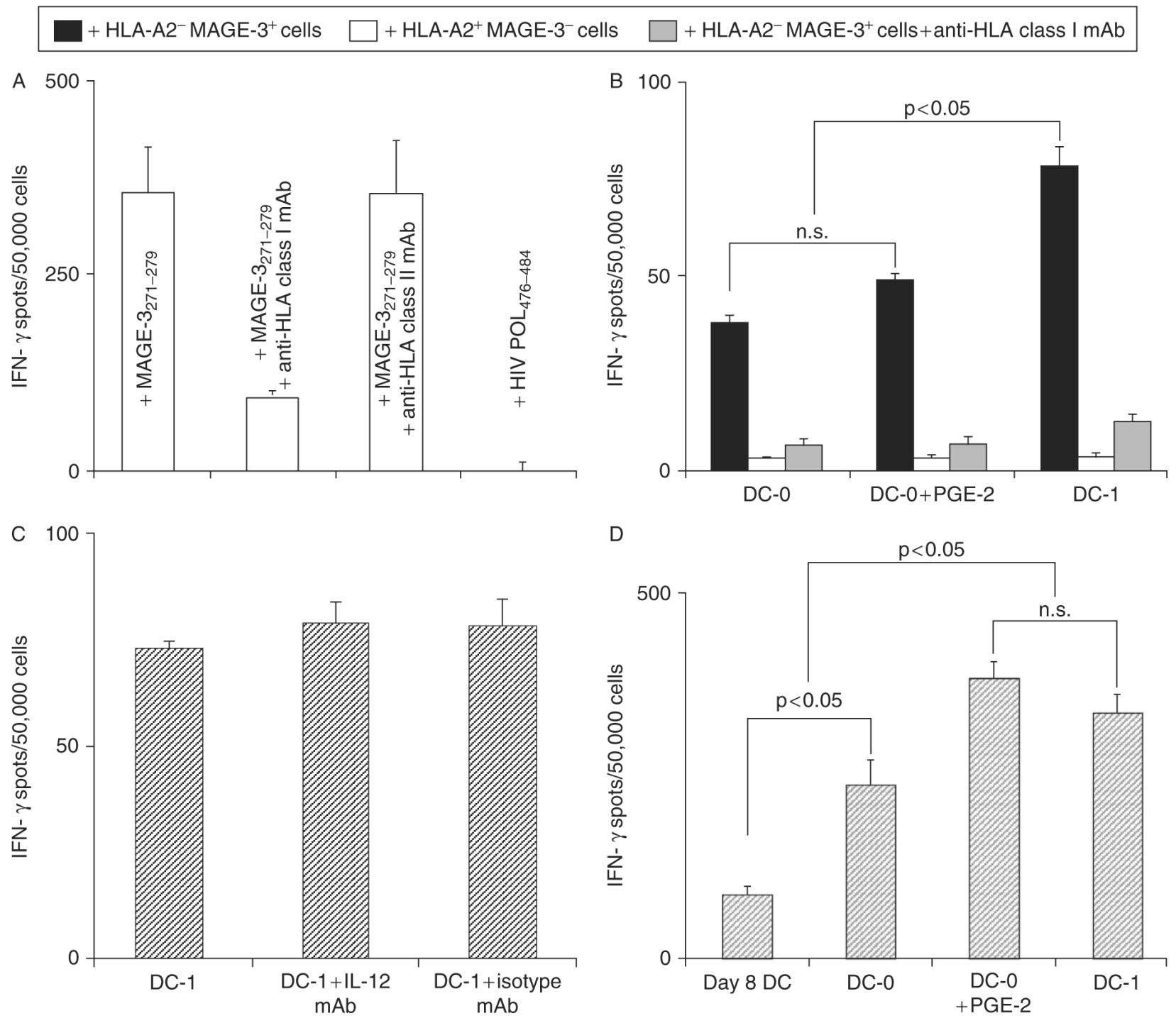

FIGURE 4.

A, Specificity of the HLA-A*0201-MAGE-3 $271-279$ specific CTL generated by in vitro stimulation. Naive CTL were stimulated as detailed in the Materials and Methods section and tested for their specificity using the TAP1/TAP2 deficient T2 cells pulsed with MAGE-3 ${ }_{271-279}$ or HIV POL $476-484$ exogenous peptides. The HLA class I specific mAb W6/32 and the HLA-DR specific mAb L243 were used as controls for determination of class I restricted activity. B, Cross-presentation of MAGE-3 $271-279$ derived from HLAA*0201- ${ }^{-}$JHU-029 cells to MAGE-3 $3271-279^{-}$-specific effector cells by DC treated with different maturation conditions. On day 6 of the DC cultures, UV-B irradiated HLA$\mathrm{A}^{*} 0201^{-}, \mathrm{MAGE}-3^{+} \mathrm{JHU}-029$ cells were added to the DC cultures, in the presence of each cytokine maturation combination, at $37^{\circ} \mathrm{C}$ for 48 hour. DC were used as targets in IFN- $\gamma$ ELISPOT assays using HLA-A*0201-MAGE-3217-279-specific CTL. The HLA class I antigen restricted CTL activity was monitored by incubating targets with HLA-A, HLA-B, HLA-C antigen-specific mAb W6/32 (gray bars). CTL were tested for specificity (as previously detailed) on every experiment where DC was used as targets (ns $=$ non significant, $P<0.05$ 1-tail permutation test). C, Apoptotic JHU-029 fed DC-1 cells were incubated with an interleukin-12 specific $\mathrm{mAb}$ or an Immunoglobulin $\mathrm{G}$ isotype control and 
used as stimulators in an ELISPOT assay. D, DC treated with the different maturation cytokine combinations were harvested and incubated with exogenous MAGE-3271-279 peptide ( $10 \mu \mathrm{g} / \mathrm{mL}$ in AIM-V media for $1 \mathrm{hr}$ at room temperature). IFN- $\gamma$ ELISPOT assays were performed using the HLA-A*0201-MAGE-3271-279 specific CTL. (ns indicates non significant, $P<0.051$ tail permutation test). CTL, cytotoxic T lymphocytes; DC, dendritic cells; mAb, monoclonal antibodies. 


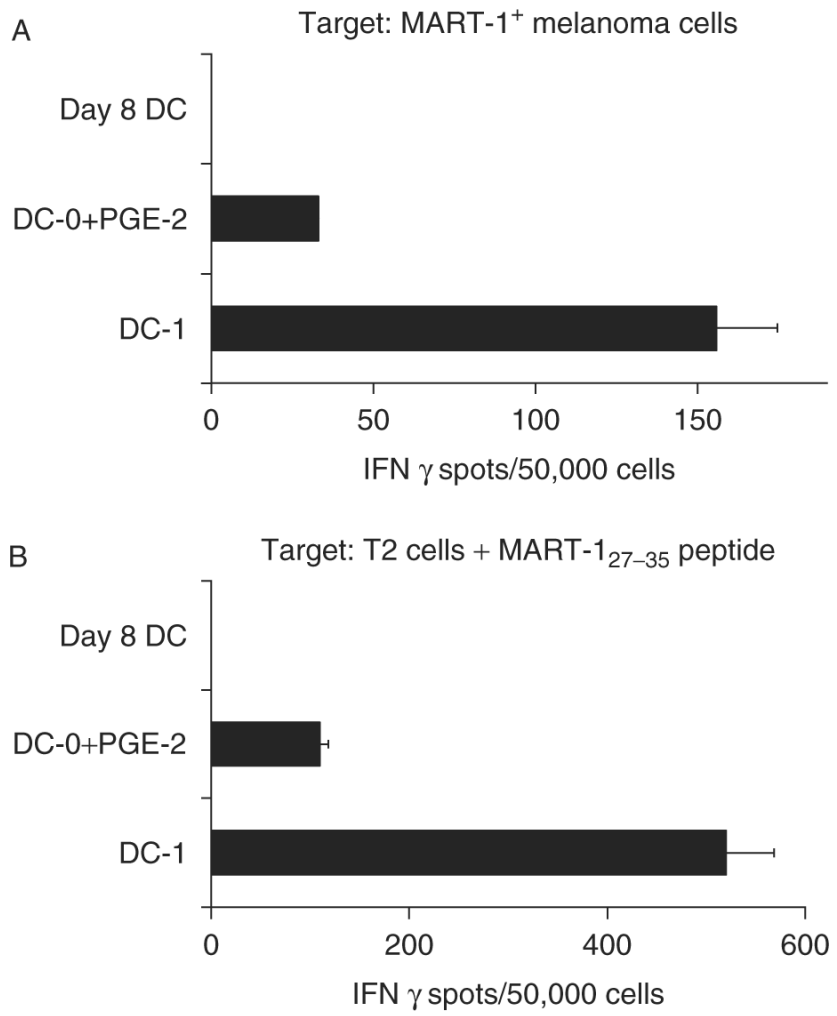

FIGURE 5.

Cross-presentation of melanoma cell-derived MART- 1 to CTL precursors by DC- 1 cells leads to improved induction of melanoma-specific CTL response. DC-1, DC-0+PGE-2 or immature DC were cultured from a healthy HLA-A2 ${ }^{+}$donor and cultured with UV-treated apoptotic FEM-X melanoma cells for 48 hour at $37^{\circ} \mathrm{C}$ as a source of MART-1 antigen. These DC were then used to sensitize autologous $\mathrm{CD}^{+} \mathrm{T}$ cells and frequencies of IFN- $\gamma$ producing melanoma antigen specific CTL were determined by ELISPOT. A, IFN- $\gamma$ spots produced by DC-primed CTL recognizing the HLA-A2 ${ }^{+}$FEM-X melanoma cell targets. B, IFN- $\gamma$ producing antigen specific CTL responding to HLA-A2 $2^{+} \mathrm{DR} 4^{+} \mathrm{T} 2$ target cells pulsed with the HLA class-I restricted melanoma peptide MART- $1_{27-35}$. CTL indicates cytotoxic T lymphocyte; DC, dendritic cell FEM-X; IFN, interferon; HLA, human leukocyte antigen. 


\section{TABLE 1}

Cytokine Dendritic Cell Cytokine Combinations Used

\begin{tabular}{lcc}
\hline & \multicolumn{2}{c}{ Cytokine Combination (Concentration/mL) } \\
\cline { 2 - 3 } & Day 8 DC IL-4/GM-CSF & 1000 IU Each for 8 d \\
\hline IL-1B & $25 \mathrm{ng}$ & $5000 \mathrm{IU}$ \\
IL-6 & $25 \mathrm{ng}$ & $3750 \mathrm{IU}$ \\
TNF- $\alpha$ & $50 \mathrm{ng}$ & $5500 \mathrm{IU}$ \\
& DC-0+PGE-2 & \\
IL-1B & $25 \mathrm{ng}$ & $5000 \mathrm{IU}$ \\
IL-6 & $25 \mathrm{ng}$ & $3750 \mathrm{IU}$ \\
TNF- $\alpha$ & $50 \mathrm{ng}$ & $5500 \mathrm{IU}$ \\
PGE-2 & $1 \mathrm{mM}$ & \\
& DC-1 & \\
IL-1B & $25 \mathrm{ng}$ & $5000 \mathrm{IU}$ \\
Poly I:C & $25 \mathrm{ng}$ & $3750 \mathrm{IU}$ \\
TNF- $\alpha$ & $50 \mathrm{ng}$ & $5500 \mathrm{IU}$ \\
IFN- $\gamma$ & - & $1000 \mathrm{IU}$ \\
IFN- $\alpha$ & - & $3000 \mathrm{IU}$ \\
\hline
\end{tabular}

DC indicates dendritic cell; IL, interleukin; IFN, interferon; PGE-2, prostaglandin-2; GM-CSF, granulocyte macrophage colony stimulating factor; TNF- $\alpha$, tumor necrosis factor- $\alpha$. 


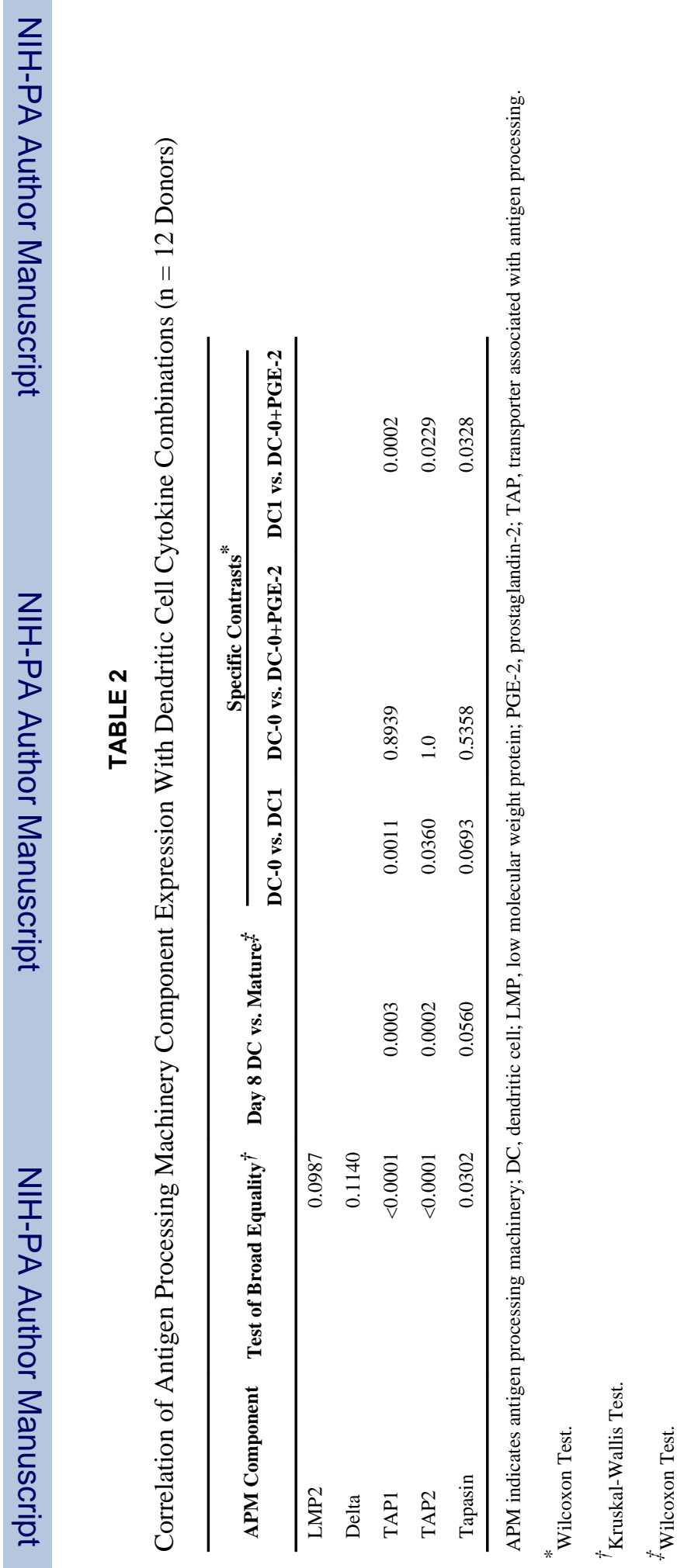

J Immunother. Author manuscript; available in PMC 2010 August 2. 